\title{
¿POR QUÉ SUBCONTRATAN LAS EMPRESAS MINERAS EN CHILE?
}

\section{Patricio Pérez y Pablo Villalobos}

Los datos disponibles muestran que el fenómeno de la subcontratación ha cobrado fuerza al interior de las empresas, caracterizando la evolución del mercado del trabajo en los últimos años. La minería no ha estado ajena a este fenómeno. A mediados de los años noventa por cada trabajador propio había 0,68 trabajadores contratistas, mientras una década después la relación es de casi dos trabajadores externos por cada trabajador propio.

Este trabajo analiza las causas de la subcontratación en la minería del cobre en Chile. Se usan datos de panel para 18 faenas en el período 2003-2008. Se concluye que los precios, las presiones de costos y el tamaño de la planta son importantes para explicar los niveles de subcontratación.

Palabras clave: subcontratación; mercado del trabajo; minería; tamaño de la planta.

Clasificación JEL: L22, L24, L72.

Patricio Pérez Oportus. Magister en Economía, Pontificia Universidad Católica de Chile. Economista de la Comisión Chilena del Cobre (Cochilco). Académico de la Facultad de Ciencias Económicas y Administrativas de la Universidad de Valparaíso. E-mail: pperez@cochilco.cl.

Pablo Villalobos Dintrans. Magister en Economía, Pontificia Universidad Católica de Chile. Economista Ministerio de Economía. E-mail: pvillalobos@economia.cl. 


\section{Introducción}

L

as cifras disponibles muestran que el fenómeno de la subcontratación ha cobrado fuerza al interior de las empresas, caracterizando la evolución del mercado del trabajo en los últimos años. Según datos del Ministerio del Trabajo ${ }^{1}$, cerca de 1 millón 200 mil trabajadores, esto es alrededor del $35 \%$ de la fuerza laboral, no son contratados directamente por las empresas principales, sino que prestan servicios a través de la subcontratación y el suministro. La minería no ha estado ajena a este fenómeno. A mediados de los años noventa alrededor del $40 \%$ de la fuerza total empleada en el sector era externo, mientras en 2008 esta proporción se había incrementado a un 65\%. Es decir, si en 1996 por cada trabajador propio había 0,68 trabajadores contratistas ${ }^{2}$, una década después había casi dos trabajadores externos por cada trabajador propio (Tabla $\mathrm{N}^{\circ} 1$ ).

Desde el punto de vista de la gestión, existe consenso en que la subcontratación es una herramienta poderosa. La evidencia señala que las empresas que optan por ella consiguen mayor flexibilidad, lo que les permite responder de mejor manera a las variaciones del entorno productivo en que operan y lograr un considerable ahorro en costos, facilitándoles la posibilidad de enfocarse solamente en el giro de su propio negocio (Teece, 1986; Alexander y Young, 1996; Abraham y Taylor, 1996; Bryce y Useem, 1998). El resultado es una fuerte inyección de eficiencia y dinamismo, que hace que las compañías sean más competitivas en el mercado en que se desenvuelven. Por ejemplo, Bryce y Useem (1998) señalan que las compañías pueden ver incrementado el valor de su marca como resultado de la mayor competitividad entregada por la subcontratación. Otro fenómeno virtuoso derivado del proceso de externalización de actividades se refiere al nacimiento de nuevas empresas, las que, en la medida que crecen en especialización y tecnología, también lo hacen en cuanto a generación de nuevos puestos de trabajo, lo que disminuye las tasas de desempleo de las zonas contiguas, fomenta la aparición de nuevos servicios y dinamiza el comercio local. Asimismo, según Silva (2007), la subcontratación ha contribuido a elevar la productividad y la competitividad de la economía en su conjunto, lo que ha reforzado el empleo.

\footnotetext{
${ }^{1}$ Véase en http://www.trabajo.gob.cl/Subcontratacion/externalizacion.htm.

${ }^{2}$ Incluye contratistas de operación minera, inversión y de servicios de apoyo y mantención.
} 
TABLA N ${ }^{\circ}$ 1: $\quad$ EVOLUCIÓN TRABAJADORES SUBCONTRATISTAS EN MINERÍA

Promedio anual de trabajadores. Período 1996-2008

\begin{tabular}{lccccc}
\hline Año & Propios & Contratistas & Total & $\begin{array}{c}\text { Razón } \\
\text { Subc./Total }\end{array}$ & $\begin{array}{c}\text { Razón } \\
\text { Subc./Propios }\end{array}$ \\
\hline & & & & & \\
1996 & 51.166 & 34.737 & 85.903 & 0,40 & 0,68 \\
1997 & 51.294 & 41.976 & 93.270 & 0,45 & 0,82 \\
1998 & 48.839 & 47.738 & 96.577 & 0,49 & 0,98 \\
1999 & 46.186 & 38.031 & 84.217 & 0,45 & 0,82 \\
2000 & 46.621 & 39.476 & 86.097 & 0,46 & 0,85 \\
2001 & 44.793 & 48.418 & 93.211 & 0,52 & 1,08 \\
2002 & 45.056 & 54.633 & 99.689 & 0,55 & 1,21 \\
2003 & 42.457 & 56.462 & 98.919 & 0,57 & 1,33 \\
2004 & 44.341 & 68.155 & 112.496 & 0,61 & 1,54 \\
2005 & 48.102 & 86.018 & 134.120 & 0,64 & 1.79 \\
2006 & 47.993 & 86.392 & 134.385 & 0,64 & 1,80 \\
2007 & 54.743 & 101.128 & 155.871 & 0,65 & 1,85 \\
2008 & 58.567 & 108.942 & 167.509 & 0,65 & 1,86 \\
\hline
\end{tabular}

Fuente: Servicio Nacional de Geología y Minería (Sernageomin).

Sin embargo, la subcontratación también trae consigo algunos costos. A juicio de Echeverría (1997), el término contratista puede tener una connotación negativa si ésta alude a una relación laboral o tipo de trabajo de segundo orden, poco calificado y precario. Además, en muchos casos, el término subcontratación suele utilizarse para referirse a labores menores, inespecíficas y con un importante componente de actividad manual o esfuerzo físico. Echeverría et al. (2001) agregan que en muchos casos la subcontratación puede ser sinónimo de atributos negativos si es que ésta es entendida como una forma de precarizar los empleos y/o de simular una relación laboral. Escobar y López (1996), por su parte, plantean que la subcontratación también dificulta la asociación sindical, ya que excluye a los trabajadores externos de la negociación colectiva y afecta la estabilidad laboral, pues suele tener naturaleza temporal, eventual o rotativa. En este último caso, agregan los autores, la subcontratación actúa como sustituto del trabajo formal o de duración indefinida, dando como resultado un trabajo no sólo más precario sino también más desprotegido.

Es importante analizar y discutir la evidencia empírica que explica los orígenes de este fenómeno, dado que existen beneficios y costos 
ligados a ella. No hay consenso en si las actuales cifras son buenas, malas o simplemente han sido el resultado hacia la evolución de un mercado del trabajo eficiente y armónico con un mundo globalizado y cambiante.

Este trabajo se plantea como objetivo buscar una explicación a las cifras de subcontratación observadas en la industria minera en los últimos años. Haciendo uso del instrumental económico disponible, se pretende identificar los posibles determinantes de la subcontratación en la industria chilena del cobre que faciliten una mejor comprensión del tema.

Además de esta introducción, el documento está organizado en seis secciones. La segunda parte revisa la forma en que la literatura ha abordado el tema, centrándose en la descripción de las variables mencionadas como los determinantes tradicionales de la subcontratación. La tercera sección hace uso de herramientas gráficas y del análisis económico para explicar las causas y beneficios de la subcontratación. La cuarta sección describe los datos y las variables usadas en el estudio, mientras que la quinta presenta la metodología de estimación, el análisis empírico y los resultados. Finalmente, la sexta sección está reservada para las conclusiones del trabajo.

\section{Determinantes de la subcontratación}

A partir del trabajo de Coase (1937) sobre la naturaleza de la firma se inicia la discusión respecto de la disyuntiva de "comprar o hacer" a la que se ven enfrentadas las empresas en su actuar diario; esta discusión ha dado lugar a una extensa literatura sobre el tema de la integración vertical. En su teoría, Coase indica que la decisión de las firmas finalmente depende de los costos relativos de usar el mercado (comprar) u organizarse al interior de la firma (hacer). Si bien la literatura identifica una gran cantidad de posibles determinantes de la integración vertical o subcontratación, estos factores pueden ser agrupados en cuatro grandes grupos: especificidad, incertidumbre, tamaño y costos laborales.

\section{a) Especificidad}

La presencia de costos de transacción da un marco para entender estas decisiones al interior de las empresas, ya que son precisamente 
estos costos los que determinan la decisión de integrarse verticalmente o subcontratar. Esta teoría, desarrollada principalmente a partir de los trabajos de Williamson $(1971,1975,1979,1985)$ y Klein et al. (1978), y extendida luego por numerosos autores ${ }^{3}$, establece que estos costos surgen a partir de una relación entre dos empresas, la cual incluye inversiones en activos específicos. La especificidad, unida a la imposibilidad de hacer contratos completos ${ }^{4}$ (Grossman y Hart, 1986) entre las firmas mandantes y las subcontratadas genera incentivos para la aparición de comportamientos oportunistas (problema del hold up) por parte de las firmas externas, los que se agudizan a medida que aumenta la especificidad de la inversión y, por lo tanto, la relación de dependencia. Este riesgo de "aprovechamiento" es el que eleva los costos de usar el mercado (subcontratar). Se espera entonces que exista una relación negativa entre la inversión en activos específicos (o la intensidad del problema de hold-up) y el nivel de subcontratación.

\section{b) Incertidumbre}

Un segundo determinante comúnmente citado en la literatura es la incertidumbre. El rol de ésta se puede ver reflejado a través de distintas formas en las decisiones de integración vertical/subcontratación de las empresas. Por una parte, puede ser entendida como un riesgo extra que se añade al riesgo natural de cada negocio, debido a una mayor varianza de los escenarios posibles. Por ejemplo, tal como argumentan Hanson (1995) y Silva (2007), las firmas podrían aumentar sus niveles de subcontratación como una forma de compartir parte de este riesgo. Este efecto se refuerza si pensamos que la subcontratación también ayudaría a reducir los costos asociados de situaciones extremas, como puede ser el cierre de la empresa. Estos factores implican un efecto positivo de la incertidumbre sobre la subcontratación. Por su parte, Abraham (1988) examina el impacto de la variabilidad de la demanda y de la variación estocástica de la oferta de trabajo de los empleados regulares sobre el uso de trabajadores externos. En esencia, este último supone que la existencia de un componente estocástico en los contratos de largo plazo de la

\footnotetext{
${ }^{3}$ Véase Gibbons (2005).

${ }^{4}$ Contratos que contemplan todas las posibles contingencias (estados de la naturaleza). Véase Joscow (2005).
} 
oferta de trabajadores propios (bonos, vacaciones, licencias, etc.) incentiva a las empresas a emplear mano de obra flexible. Usando los datos de una encuesta a más de 400 empresas americanas, la autora concluye que existe una relación positiva entre el uso de mano de obra flexible y fluctuaciones estacionales y cíclicas de la demanda. Sus resultados también indican que las empresas, además de usar la subcontratación por cuestiones derivadas de la especialización y como una vía para enfrentar las fluctuaciones en los niveles de trabajo, lo hacen como un mecanismo que les permite reducir los costos. Las relaciones anteriores han sido testeadas por Abraham y Taylor (1996) y González et al. (2000), quienes confirman la existencia de un efecto positivo entre las fluctuaciones de oferta y demanda y el nivel de subcontratación.

Por otra parte, una mayor incertidumbre aumenta los costos (dificulta) de establecer acciones para cada posible contingencia (existen más estados posibles), lo que en un modelo de principal-agente - como el que describe la relación entre una firma mandante y una subcontratada - no permite identificar si un mal resultado es producto del comportamiento del agente o culpa del azar. Lo anterior lleva a que, para cierto nivel de especificidad, un mayor grado de incertidumbre aumente el problema del hold-up, incrementando los costos de subcontratar. De modo que el efecto combinado de ambas variables y la subcontratación debiera ser negativo, por lo que en presencia de incertidumbre las firmas deberían disminuir el nivel de mano de obra externa.

En resumen, el efecto de la incertidumbre es incierto, ya que existen fuerzas positivas y negativas que influyen en los niveles de subcontratación, por lo que el resultado final dependerá de cuáles predominen.

\section{c) Tamaño}

Otro grupo de variables analizadas frecuentemente en la literatura se relaciona con el tamaño de la firma. La idea de división del trabajo y especialización se desprende del trabajo de Adam Smith y fue vinculada con la teoría de la integración vertical a partir del trabajo de Stigler (1951). La intuición señala que a medida que una firma aumenta de tamaño, necesita ir dejando de lado algunas tareas no centrales de su 
negocio, de modo de ir concentrándose en las que son más específicas a su giro empresarial. Se esperaría, por lo tanto, un mayor nivel de subcontratación en la medida que el tamaño o el crecimiento de la firma sea mayor.

Unido a esta posibilidad de expandir el mercado de la firma se encuentran otras variables que surgen a partir de ella, como la dispersión geográfica y la capacidad de monitoreo. Ambos factores refuerzan el efecto del tamaño sobre la subcontratación. Por un lado, se espera que a medida que aumente el tamaño de la firma, se extienda su mercado y con ello necesite expandir su cobertura geográfica. En presencia de costos de transporte, la opción de tener una empresa muy disgregada (i.e., que realice tareas en distintos puntos) puede ser demasiado costosa. De esta forma, la dispersión geográfica tiende a disminuir el costo relativo de usar el mercado y, con ello, se generan incentivos para que la empresa incremente el nivel de subcontratación. Por otro lado, una empresa muy grande tiene mayores dificultades para monitorear la labor de los trabajadores propios, por lo que, en este caso, resultará más conveniente subcontratar parte de las tareas de producción, monitoreando solamente su resultado final.

Davis-Blake y Uzzi (1993), utilizando los datos de una encuesta a empleadores americanos realizada por el U. S. Department of Labor (Departamento del Trabajo de Estados Unidos), analizan la influencia de distintos factores sobre el uso de contratistas independientes ${ }^{5}$. Concluyeron que el tamaño de la firma, junto a otras variables, tales como las variaciones en el empleo, la dispersión de plantas y la burocratización, tendría un efecto positivo sobre los niveles de subcontratación.

\section{d) Costos laborales}

Una última variable que puede estar tras las decisiones de subcontratación tiene que ver con los costos laborales. Los trabajos de Abraham y Taylor (1996) y Gramm y Schnell (2001) postulan que en algunos casos el uso de dotaciones de mano de obra flexible, en lugar de trabajadores propios, permite reducir costos laborales, principalmente a través del ahorro en términos de salario y otros beneficios. Por su parte, rales.

\footnotetext{
${ }^{5}$ En el mismo estudio también analizan el efecto sobre los trabajadores tempo-
} 
Houseman (1997), usando los resultados de una encuesta para empresas americanas, encuentra evidencia significativa de que las firmas que ofrecen atractivos beneficios a sus trabajadores regulares tienden a intensificar el uso de mano de obra flexible. Una posible explicación para este fenómeno, según el autor, sería que los empleadores están dispuestos a ofrecer beneficios atractivos (vacaciones y licencias pagadas, seguros de salud, bonos, etc.) sólo a un segmento definido de su fuerza de trabajo, pues legalmente no pueden discriminar, en términos de beneficios a asignar, entre trabajadores propios. Así, por medio de este ahorro, los empleadores pueden retener a sus mejores trabajadores, por lo que tienden a intensificar el uso de mano de obra flexible.

En esta línea se esperaría, entonces, que mientras más altos sean los salarios y beneficios que perciben los trabajadores propios, mayor sea el incentivo a subcontratar. De la misma forma, mientras mayor es el número de trabajadores propios de una firma, menores incentivos existirán para incrementar la dotación de trabajadores regulares.

\section{Un análisis económico para la subcontratación en la minería}

\subsection{Modelo inicial}

Supongamos una firma que produce un único bien homogéneo utilizando dos factores, capital (K) y trabajo (L), y que este bien puede ser producido indistintamente por trabajadores propios o externos. Supongamos también que en el corto plazo el stock de capital está fijo, que existen rendimientos decrecientes de los factores y que la demanda es relativamente inelástica. La industria minera se ajusta a estas características, ya que las condiciones del yacimiento exigen que el diseño de la mina y la tasa de procesamiento del mineral sean planificados de antemano; lo anterior impone ciertas rigideces por el lado de la demanda de mano de obra. La oferta por su parte, es relativamente elástica y bien comportada.

La Figura $\mathrm{N}^{\circ} 1$ ilustra este caso, donde la curva $\mathrm{VPM}_{\mathrm{L}}$ denota el valor del producto marginal del factor trabajo ${ }^{6}$. En ausencia de distorsiones, el equilibrio de mercado se encontraría en el punto E, con un

${ }^{6}$ Se entiende como el valor a precio actual de mercado de la producción adicional generada por una unidad adicional del factor trabajo. 
FIGURA N ${ }^{\circ}$ 1: $\quad$ LA ECONOMÍA DE LA SUBCONTRATACIÓN

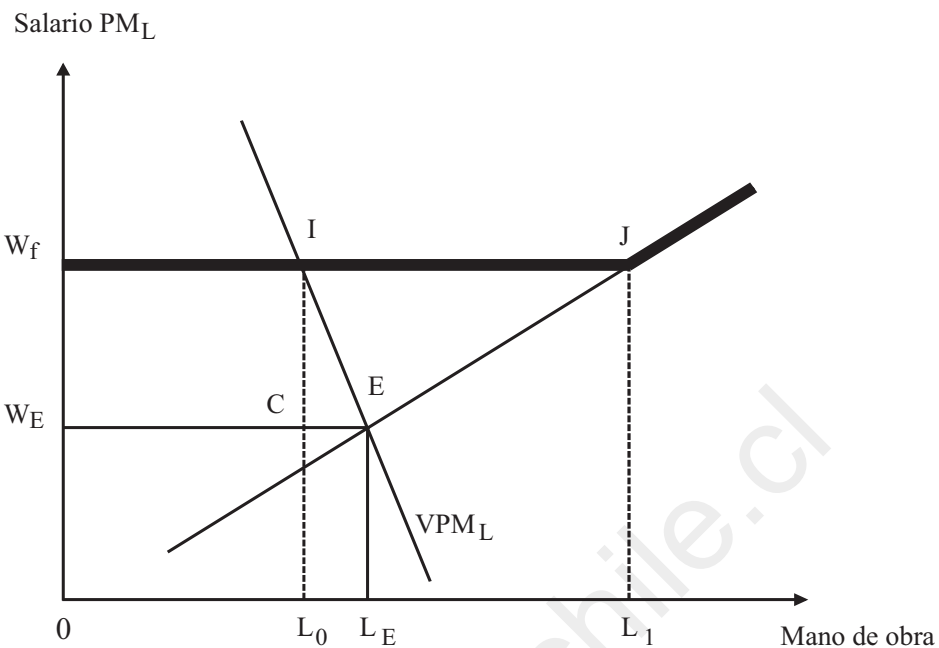

Fuente: Elaboración de los autores.

nivel de contratación $0 \mathrm{~L}_{\mathrm{E}} \mathrm{y}$ un salario pagado $\mathrm{W}_{\mathrm{E}}$. Así, el área $\mathrm{W}_{\mathrm{E}} \mathrm{EL}_{\mathrm{E}} 0$ corresponde al costo total de la mano de obra y el área sobre $\mathrm{W}_{\mathrm{E}} \mathrm{E} \mathrm{y}$ bajo la curva $\mathrm{VPM}_{\mathrm{L}}$ representa el retorno del capital. Ahora, supongamos la existencia de un sindicato que negocia con esta firma el establecimiento de un salario mínimo igual a $\mathrm{Wf}^{7}$. Bajo este esquema, la curva de oferta relevante para esta firma estará fija entre el tramo $\mathrm{W}_{\mathrm{f}} \mathrm{J}$, por lo que el nivel de contratación será solamente $0 \mathrm{~L}_{0}$ en vez de $0 \mathrm{~L}_{\mathrm{E}}$. La diferencia $\mathrm{L}_{0} \mathrm{~L}_{\mathrm{E}}$ es el desempleo que se atribuye a este poder sindical. El área del rectángulo $\mathrm{W}_{\mathrm{f}} \mathrm{ICW}_{\mathrm{E}}$ representa la transferencia que hacen los dueños del capital a los trabajadores propios, mientras que el triángulo ICE representa la pérdida social.

${ }^{7}$ En este caso lo importante es la existencia de diferencias en los costos laborales de ambos grupos de trabajadores (propios y subcontratistas), sean éstas generadas por diferencias en los salarios o por otro tipo de beneficios; a fin de cuentas el eje vertical está midiendo el precio del trabajo, que para el caso del empleador y del trabajador es más que el salario. El origen de las diferencias tampoco es relevante, sean éstas producidas por la existencia de un sindicato o por otra razón. 


\subsection{Ganancias de la subcontratación}

El análisis anterior muestra con claridad que el aumento de salarios lleva a un equilibrio socialmente ineficiente. En este esquema, la subcontratación puede ser usada para alcanzar una solución óptima. Si partimos del nuevo equilibrio en $\mathrm{I}$, con un salario $\mathrm{W}_{\mathrm{f}} \mathrm{y}$ un nivel de contratación $\mathrm{L}_{0}$, se puede ofrecer un salario distinto, como $\mathrm{W}_{\mathrm{E}}$, y contratar trabajadores hasta el punto $\mathrm{L}_{\mathrm{E}}$. De esta forma, en un mundo donde la subcontratación es posible, coexisten dos tipos diferentes de trabajadores - propios y subcontratados - con dos salarios distintos. La situación, más allá de las posibles consideraciones morales, es eficiente, ya que permite crear nuevos puestos laborales $\mathrm{L}_{\mathrm{E}}-\mathrm{L}_{0}$, y reducir (y en el extremo eliminar) el desempleo y la pérdida social a causa de la rigidez. De hecho, todos los trabajadores pueden trabajar por un salario $\mathrm{W}_{\mathrm{E}}$. Lo anterior permite, entonces, que la firma alcance el stock de mano de obra óptimo $\overline{0 \mathrm{~L}_{\mathrm{E}}}$, con lo que el área de triángulo ICE representaría la ganancia neta de la subcontratación.

\subsection{Efectos de un shock}

Tomando como referencia el modelo anterior, supongamos ahora que aumenta la demanda por el bien que esta firma produce (Figura $\mathrm{N}^{\circ} 2$ ). Si asumimos que ésta es una demanda relacionada de la demanda de trabajo, entonces veremos una expansión de la curva $\mathrm{VPM}_{\mathrm{L}}$ a $\mathrm{VPM}_{\mathrm{L}}{ }^{\prime}$. En ausencia de distorsiones, el equilibrio se encontraría en E' para el nivel de renta $\mathrm{W}_{\mathrm{E}}{ }^{\prime}$, con un nivel de contratación óptimo igual a $0 \mathrm{~L}_{\mathrm{E}}$.

Si el shock es permanente, el óptimo de contratación de dotación propia se daría en $\overline{\mathrm{OL}_{2}}$, bajo el punto I', de modo que la diferencia $\overline{0 \mathrm{~L}_{\mathrm{E}}}-\overline{0 \mathrm{~L}_{2}}=\overline{\mathrm{L}_{2} \mathrm{~L}_{\mathrm{E}}^{\prime}}$ indicaría la cantidad de mano de obra que las compañías estarían supliendo con mano de obra externa. En este caso, el cambio permanente en la demanda puede verse como un crecimiento en el tamaño de la firma. Para que este cambio de tamaño genere un cambio más que proporcional en la cantidad de subcontratados, es decir, que aumente la proporción del total de trabajadores que son subcontratados, se requieren condiciones especiales, como por ejemplo que la curva 


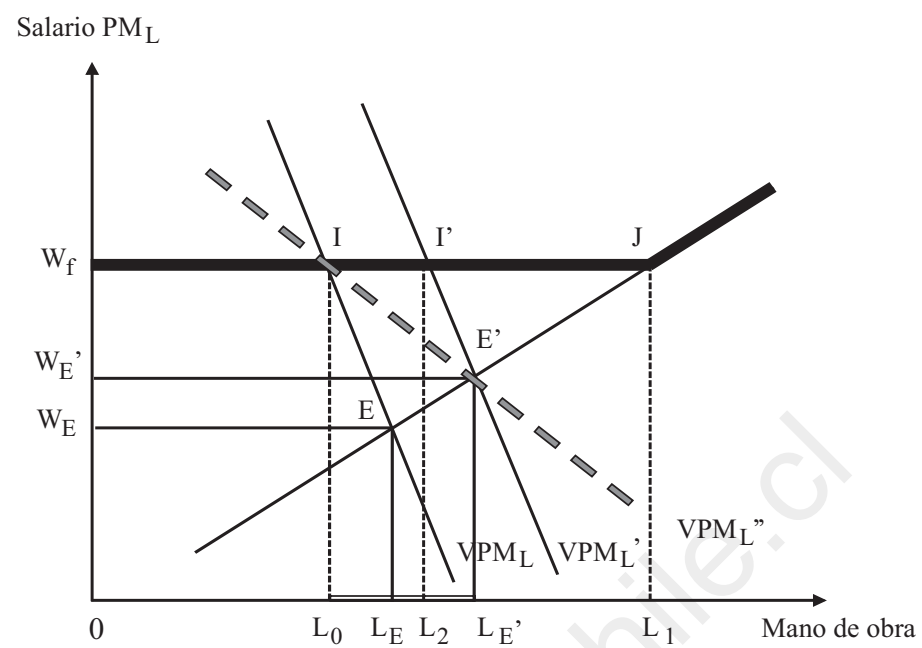

Fuente: Elaboración de los autores.

$\mathrm{VPM}_{\mathrm{L}}$ ' cambie su pendiente (disminuya la pendiente en valor absoluto), lo que se consigue si el shock se manifiesta de manera no neutral ${ }^{8}$.

Por otra parte, si el cambio es transitorio, como es el caso de la mayoría de los shocks en la minería, la curva relevante pasa a ser $\mathrm{VPM}_{\mathrm{L}}$ " (línea punteada) y toda la diferencia, vale decir, el tramo $\mathrm{L}_{0} \mathrm{~L}_{\mathrm{E}}$, $=0 \mathrm{~L}_{\mathrm{E}}-0 \mathrm{~L}_{0}$ sería cubierto con mano de obra externa. El salario pagado para este grupo de trabajadores externos sería $\mathrm{W}_{\mathrm{E}}$, levemente superior a $\mathrm{W}_{\mathrm{E}}$ como resultado del aumento de la demanda. Este caso refleja el comportamiento de las firmas para ajustar cambios temporales y muestra claramente que aumenta la proporción de subcontratados al interior de ellas.

El principal aporte de este análisis es que logra, con un modelo simple, incluir tres de los cuatro factores antes mencionados para explicar las decisiones de subcontratación. A través de un escenario inicial donde existen diferencias en costos laborales, se muestra el efecto tanto de cambios en tamaños de las firmas como de ajustes a shocks tempo-

${ }^{8}$ Por ejemplo, en el largo plazo, si el shock se mantiene, la demanda derivada por trabajo podría volverse más elástica, en la medida que existen más sustitutos disponibles (e.g. trabajadores de características equivalentes). 
rales en las decisiones de contratación de mano de obra. Una segunda ventaja es que los supuestos se ajustan a la industria de la minería, que es la analizada en este trabajo. Finalmente, es posible apreciar tanto los pros como los contras de la subcontratación: por un lado permite ganancias a las empresas a través de una mayor flexibilidad, mientras que por otro genera condiciones de trabajo desiguales para distintos trabajadores, lo que para algunos autores tiene costos tanto para los trabajadores por la vía de precarizar los empleos (Echeverría, 1997; Escobar y López, 1996; Echeverría et al., 2001), como para las compañías debido al surgimiento de los conflictos y huelgas (Daroch, 2008; López, 2008).

\section{Los datos}

\subsection{Una mirada al sector minero}

Para el análisis de la subcontratación usamos datos de panel de 18 faenas mineras para el período 2003-2008, los que se obtienen de distintas fuentes. En la Tabla A1 del Anexo se entrega el detalle de las faenas seleccionadas y su nivel de producción. Considerar a estas 18 firmas supone incluir las operaciones mineras que en conjunto han aportado más del $90 \%$ de la producción chilena de cobre en la última década (Tabla $\mathrm{N}^{\circ} 2$ ). Por último, el panel no es balanceado, esto porque la producción de Spence sólo comenzó en el año 2006.

En la Tabla A2 del Anexo se presentan las estadísticas descriptivas de la muestra. Se desprende que, en promedio, las faenas tienen alrededor de 3.100 trabajadores externos, que el $75 \%$ de la muestra tiene hasta 4.200 trabajadores bajo esta modalidad de contrato y que las faenas que más subcontratan alcanzaron hasta 11.600 trabajadores. En cuanto a la proporción de trabajadores externos sobre el total de dotación, en promedio es cercana al $65 \%$, es decir, cada faena tiene en promedio 2,7 trabajadores externos por cada trabajador regular o de planta contratado. También se muestra la estadística descriptiva de algunas series de costos, en particular la serie de costos brutos ${ }^{9}$ (real),

${ }^{9}$ Siguiendo la nomenclatura del Banco Mundial, se utiliza el concepto de "costo bruto" para definir aquellos costos que incluyen los costos operacionales directos, indirectos, depreciación y cargos por intereses, sin descontar los créditos por venta de subproductos derivados de la explotación. 
TABLA N ${ }^{\circ}$ 2: $\quad$ PARTICIPACIÓN DE LAS FAENAS SELECCIONADAS EN LA PRODUCCIÓN TOTAL DE CHILE

(Período 2000-2008, en miles de tons. métricas de fino, MTMf)

\begin{tabular}{lccc}
\hline Año & $\begin{array}{c}\text { Producción total } \\
\text { faenas seleccionadas }\end{array}$ & $\begin{array}{c}\text { Producción chilena } \\
\text { total }\end{array}$ & $\begin{array}{c}\text { Participación } \\
(\%)\end{array}$ \\
\hline & & & \\
2000 & $4.426,3$ & $4.602,0$ & $96 \%$ \\
2001 & $4.557,4$ & $4.739,0$ & $96 \%$ \\
2003 & $4.419,5$ & $4.580,6$ & $96 \%$ \\
2004 & $4.721,6$ & $4.904,2$ & $96 \%$ \\
2005 & $5.198,7$ & $5.412,5$ & $96 \%$ \\
2006 & $5.079,4$ & $5.360,8$ & $95 \%$ \\
2007 & $5.095,9$ & $5.360,8$ & $95 \%$ \\
2008 & $5.272,2$ & $5.557,0$ & $95 \%$ \\
& $4.925,6$ & $5.330,3$ & $92 \%$ \\
\hline
\end{tabular}

Fuente: Cochilco: “Anuario de Estadísticas del Cobre y Otros Minerales (1990-2009)”, 2010.

cash $\operatorname{cost}^{10}(\mathrm{C} 1)$ y de costo neto a cátodo ${ }^{11}(\mathrm{C} 3)$. Se observa que en el período 2003-2008 los costos brutos promediaron 92,62 ф/libra, con un máximo de 195,01 centavos. Los cash cost, por su parte, registraron un promedio de 53,48 ф/libra.

La diferencia entre ambos costos se explica por la incidencia de los créditos por subproductos, los que contribuyen a relajar los costos brutos de manera considerable. En efecto, para el caso de los cash cost, el máximo en el período fue un promedio de 100,14 ф/libra, esto es, más de 90 centavos por debajo del máximo registrado en los costos brutos.

Asimismo, interesante resulta observar que el $75 \%$ de las faenas nacionales se ubica en un rango de cash cost inferior a los 66,22 centavos (dólar/libra). Los costos netos a cátodo (C3), por su parte, promediaron 78,64 centavos (dólar/libra en el período), con un máximo de 152,16 ф/libra. Para el análisis se utilizó esta última serie (C3), básicamente porque consideramos que refleja de mejor manera la estructura de costos de la compañía (incluye costos operacionales y no operacionales) y porque a nuestro juicio resulta fundamental incluir los descuentos por subproductos, ya que éstos son un componente importante en la estructura productiva de las compañías y en muchos casos marcan la diferencia entre ejecutar o no ejecutar un proyecto minero.

\footnotetext{
${ }^{10}$ Incluye los costos operacionales directos más los créditos por subproductos.

${ }^{11}$ Incluye el cash cost (C1) más la depreciación, costos indirectos e intereses.
} 
La serie de producción muestra que, en promedio, las faenas nacionales tuvieron un nivel de producción entre 2003 y 2008 de 291,34 MTM de cobre fino, con un máximo de 1.483,9 MTM $^{12}$; mientras que la ley fue de $0,99 \%$, con un máximo de $1,74 \%$ y que sólo un $25 \%$ de ellas presentó una ley superior a $1,11 \%$.

En cuanto a los salarios, se desprende que la renta real promedio pagada a los trabajadores propios (en términos de centavos de dólar por libra) supera en más de $65 \%$ el promedio de la renta de los subcontratistas $(13,66 v s$. 8,24); además se observa que mientras el $75 \%$ de los trabajadores propios recibe rentas superiores a los 9,33 $\notin /$ libra, la de los trabajadores externos apenas se empina por los 2,58 $\not /$ libra, vale decir, 3,7 veces inferior.

\subsection{Descripción de las variables y fuentes utilizadas}

\subsubsection{Variable dependiente}

La subcontratación se mide, a nivel de cada faena, como el porcentaje de trabajadores subcontratados sobre el total de trabajadores (SUBTOT). La serie de subcontratados y trabajadores propios se obtiene desde el Reporte de Empresas Mandantes por Categorías con Contratistas proporcionado por el Servicio Nacional de Geología y Minería (Sernageomin). Esta serie parece ser la variable más concreta de medición, ya que refleja directamente la decisión de subcontratar o tener trabajadores propios. Alternativas a esta variable usadas en otros estudios son medir el valor del total de actividades subcontratadas respecto del total de producción de la firma o el porcentaje que representan los subcontratados respecto del costo total de la mano de obra. Ambas variables no logran reflejar directamente la decisión de las firmas, ya que existen otros factores involucrados (e.g. precios) que no permiten ver el impacto sobre la variable de interés.

\subsubsection{Variables independientes}

Dentro del conjunto de variables explicativas hemos considerado aquellas que miden especificidad, incertidumbre, tamaño y costos laborales.

${ }^{12}$ Producción de Escondida en 2007, Cochilco, “Anuario de Estadísticas del Cobre y Otros Minerales, 1990-2009”, 2010. 
Especificidad. Idealmente, nos gustaría contar con información acerca de qué tipo de trabajadores (propios o subcontratados) realiza cada tarea, sin embargo esta información no está disponible. Una aproximación para captar especificidad, propuesta por González et al. (2000), es medir el uso alternativo de los activos de la firma a través del tamaño de mercado de cada producto producido. El espíritu de esta medida es que en industrias con distintos productores (e.g. sector construcción), el número de firmas produciendo cada producto (e.g. casas, puentes, edificios) es una proxy de la especificidad bajo el entendido que distintos productos requieren distintos insumos y distintos niveles de especificidad. En la minería del cobre, sin embargo, existe un único producto que es homogéneo, por lo tanto la estrategia es inútil. No obstante, se puede realizar una medición más directa de la existencia de especificidad a través del proceso de producción. En efecto, en el caso del cobre existen diferencias en los procesos productivos, los que involucran distintos niveles de conocimientos y por lo tanto distintos niveles de inversión en activos específicos. En general, el cobre es producido a través de dos tipos de procesos: flotación-fundición-electrorrefinación (sulfuros) o lixiviación-extracción solventes-electroobtención (óxidos), lo que podría considerarse como una proxy de especificidad, en la medida que el usar uno u otro proceso significa el uso de distintos activos (maquinarias, conocimientos). La idea es que la faena no es más específica a medida que se especializa en uno u otro proceso, sino en tanto que alguno de los procesos usa activos específicos en su producción. En este caso tomamos el proceso de producción vía óxido para medir este efecto. Esperamos que si el proceso de producción vía óxidos requiere del uso de más activos específicos que el proceso de sulfuros, entonces la variable mostrará un efecto negativo sobre los niveles de subcontratación.

De esta manera, para medir la especificidad se construye la variable TIPO_OXSLF, que mide el porcentaje de la producción total que es obtenido vía proceso de óxido ${ }^{13}$. Los valores de esta variable se ubican en el rango [0,1]. Estos datos se obtienen de la base de datos Brook Hunt. Como se ha señalado, el objetivo es tratar de captar diferencias en los procesos de producción que puedan dar origen a distintos niveles de uso de activos específicos en las diferentes faenas.

${ }^{13} \mathrm{Si}$ bien el proceso de sulfuros y el de óxido son dos procesos que llegan a productos distintos: concentrado y cátodos, respectivamente, la base de Brook Hunt presenta los datos de forma que sean comparables (cobre contenido o pagado). 
Incertidumbre. Para medir la incertidumbre contamos con distintas alternativas. Por una parte podemos medir variabilidad a través de cambios en el número de trabajadores (DTRAB) o en la producción (DTRATADO) de un período a otro. Con esto tratamos de determinar si una faena enfrenta cambios en su demanda que generen incentivos en su decisión de subcontratación. La serie de producción se obtiene del "Anuario de Estadísticas del Cobre y Otros Minerales", editado por Cochilco en 2010, mientras que los datos del número de trabajadores se obtienen del Reporte de Empresas Mandantes por Categorías con Contratistas proporcionado por Sernageomin. De manera alternativa se usa una estimación de la variación del precio real del cobre respecto al precio de tendencia (DPRECIOR). Para construir esta variable usamos una serie de precios del cobre desde 1850 hasta $2000^{14}$, a la que le empalmamos los precios nominales desde 2001 hasta 2008; sin embargo, dado que la serie de IPM USA está disponible desde 1913, la serie de precios real a la cual se le extrae la tendencia es a partir de este último año. Desde la base de datos de Cochilco se obtiene la serie de precio del cobre medido en dólares americanos, la que se deflacta por el índice de precios al por mayor para todos los commodities (IPM all commodities), obtenido de la base de datos de la U. S. Bureau of Labor Statistics (BLS). Se utiliza el año 2000 como referencia para el cálculo del año base. Para aislar la tendencia utilizamos el filtro de Hodrick y Prescott. Esta variable es de suma importancia, ya que capturaría las fluctuaciones de corto plazo (entre 2003 y 2008), similares a las propuestas por Abraham (1988). También como una forma de medir incertidumbre, se construye la variable (PRESIONR), definida como la diferencia entre el precio real y los costos reales de producción. La intuición de esta variable es que mientras mayores son los costos, mayor es la presión sobre el precio de esta faena, de modo que más alta es la incertidumbre y por tanto mayor debería ser el nivel de subcontratación, pues serían menores los costos de despido asociados al cierre. El componente de precio intenta capturar el efecto de variaciones de precio sobre la subcontratación. La intuición es similar a la de la variable DPRECIOR, esperando una relación positiva entre precios y subcontratación, como forma de responder a shocks de corto plazo.

${ }^{14}$ J. Díaz, et al. (2006). 
Tamaño. Como variable de tamaño tenemos la producción de cada faena en cada período de tiempo, para lo cual contamos con dos variables: producción bruta de cobre $(\mathrm{Q})$ y producción ajustada por ley $(\text { TRATADO })^{15}$. La primera refleja la cantidad de producto final obtenido (miles de toneladas de cobre fino) en la explotación de cada faena, mientras que la segunda es una aproximación al mineral tratado en planta que en sí es un indicador del esfuerzo requerido para obtener la cantidad de producto final. Usamos esta última porque el nivel de contratación de mano de obra está más ligado al esfuerzo en la producción y no al nivel de producción de cobre propiamente tal. La información sobre la ley media del mineral extraído, que sirve para realizar el ajuste al mineral tratado, se obtiene de la base de datos Brook Hunt. Para las faenas con procesos Sx-Ew se utiliza la $\mathrm{Cu}$ grade total del Heap Leach, mientras que para el resto de las faenas se utiliza la head grade o ley media de planta.

Respecto a la variable tamaño surge la inquietud de que esta variable pudiera ser endógena, es decir que nos encontremos con un caso de simultaneidad donde no sólo el tamaño determine la fracción subcontratada, sino que la fracción subcontratada también determine el tamaño de la empresa. Sin embargo, en el caso de nuestro análisis este eventual problema queda descartado. Lo anterior se explica porque, en general, las empresas de la minería del cobre no determinan el nivel de producción que les permitiría maximizar las utilidades, sino que producen lo máximo en función de su capacidad instalada. Esta capacidad viene determinada básicamente por las características del yacimiento, en particular, la riqueza, volumen y calidad de los recursos mineros. De esta forma, es posible tratar el nivel de producción de cada faena como una variable exógena, descartando el posible problema de endogeneidad.

Costos laborales. Finalmente, con el objeto de cuantificar posibles efectos de los costos laborales sobre la subcontratación, usamos salarios pagados a los trabajadores propios (WPR) y el cambio de éstos entre períodos consecutivos (DWP). También construimos la variable (RAZONW) como el cociente entre el salario real pagado a los trabajadores propios y los subcontratados. La hipótesis es que mientras más

\footnotetext{
${ }^{15}$ Se prueban distintos rezagos de la variable TRATADO.
} 
altos son los salarios pagados a los trabajadores propios, mayor es la proporción de subcontratación en la faena. Los datos de salarios pagados tanto a trabajadores propios como a contratistas están expresados en centavos de dólar por libra y son obtenidos de la base de datos Brook Hunt.

Como alternativa, utilizamos la tasa de accidentabilidad (TASA ACC) como variable de riesgo de la faena, la que se obtiene del Reporte de Empresas Mandantes por Categorías con Contratistas proporcionado por Sernageomin. La intuición es que mientras más riesgosa es la faena, ésta tiende a hacerse más intensiva en el uso de trabajadores externos, como una forma de evitar los riesgos para los trabajadores propios. $\mathrm{Si}$ bien esto no lo permite la nueva ley de subcontratación ${ }^{16}$, que rige desde el año 2007, como este documento analiza a partir del año 2003, se intentará medir si ésta fue o no una de las causas utilizadas por las empresas chilenas para subcontratar.

Adicionalmente se testea el efecto de otras variables, como la propiedad (DEP), para medir si existen diferencias en los niveles de subcontratación, provocadas por la operación de faenas por parte de una empresa pública (Codelco) y las empresas privadas. Esto, porque existe el juicio generalizado de que la empresa estatal tiende a subcontratar más que las empresas privadas. También, para medir el impacto de la entrada en vigencia de la ley de subcontratación, se usa una dummy para el año 2007 (Ley2007).

\section{Metodología y resultados}

Para testear nuestra hipótesis usamos un panel con datos de las 18 faenas mineras en el período 2003-2008.

El modelo básico para la faena i-ésima en el año t viene dado por:

$\mathrm{S}_{\mathrm{it}}=\mathrm{s}$ (Especificidad, Incertidumbre, Tamaño, Costos Laborales)

El que puede resumirse como sigue:

${ }^{16}$ Ley $\mathrm{N}^{\circ} 20.123$, que regula el trabajo en régimen de subcontratación, el funcionamiento de la empresas de servicios transitorios y el contrato de trabajo de servicios transitorios. 


$$
S_{i t}=\alpha+\beta X_{i t}^{\prime}+\lambda_{t}+\mu_{i t}
$$

Donde $S_{i t}$ muestra el nivel (grado) de subcontratación de la faena i-ésima en el período t; $X_{i t}^{\prime}$ representa el set de variables explicativas que varían entre faenas y en el tiempo descritas previamente; $\lambda_{t}$ es un set de variables dummies que reflejan variación en el tiempo; $\mu_{i t}$ corresponde al error del modelo, $\alpha$ es la constante y $\beta$ el set de parámetros a estimar.

Por otro lado, y dado que la variable dependiente SUBTOT se encuentra limitada entre 0 y 1 , necesitamos una metodología de estimación consistente con esta restricción. Una forma de abordar esto es a través de una transformación funcional logística:

$$
S_{i t}=\frac{e^{\alpha+\beta X_{i t}^{\prime}+\lambda_{t}+\mu_{i t}}}{1+e^{\alpha+\beta X_{i t}^{\prime}+\lambda_{t}+\mu_{i t}}}
$$

Reordenando y aplicando logaritmos, se llega a la siguiente expresión:

$$
\log \left(\frac{S_{i t}}{1-S_{i t}}\right)=\alpha+\beta X_{i t}^{\prime}+\lambda_{t}+\mu_{i t}
$$

Si asumimos que el error $\mu_{i t}$ es independiente de las variables explicativas, entonces el modelo puede ser estimado simplemente por mínimos cuadrados (OLS). Sin embargo, esta metodología resulta insuficiente para captar la heterogeneidad no observada que estaría influyendo sobre la variable de interés, por lo que en este caso la estimación por OLS entregaría estimadores sesgados debido a la omisión de variables relevantes.

No obstante, una de las principales ventajas de los datos de panel es que la dimensión temporal enriquece la naturaleza de los datos, siendo posible controlar por algunas de estas variables aun cuando no las observemos. Para ello asumimos que el error viene dado por la siguiente expresión:

$$
\mu_{i t}=\delta_{i}+\varepsilon_{i t}
$$


donde $\delta_{i}$ es un efecto específico a cada firma que refleja esta heterogeneidad no observada y $\varepsilon_{i t}$ es ruido blanco. El componente no observado se refiere a aquellas características que son idénticas en todas las observaciones de la misma faena y que no es posible medir. Ejemplos de esto pueden ser la capacidad de gestión, el estilo de administración o la oportunidad en la toma de decisiones.

En general, y siguiendo esta tesis, el estimador más adecuado dependerá de la naturaleza de la relación entre los efectos individuales $\left(\delta_{i}\right)$ y los regresores. Para nuestro caso, es probable que la "capacidad de gestión" esté ligada a aspectos tales como la cantidad de procesos que tiene cada faena, la dispersión de éstas, o sea, qué tan lejos se encuentra un proceso de otro, el tamaño de estos procesos, características naturales de la mina (tipo de mineral, nivel freático ${ }^{17}$, condiciones ambientales, altura, ubicación de la mina, entre otros), etc. Entonces, si los efectos individuales y los regresores están correlacionados, la mejor alternativa sería un modelo de efectos fijos. Éste toma la diferencia entre todas las variables y sus promedios sobre el tiempo, eliminando el sesgo consecuencia de la correlación, de modo que $\delta_{i}$ se mantiene fijo y no varía a través del tiempo. Ahora bien, si se asume que los efectos individuales son ortogonales a los regresores, la alternativa es asumir que $\delta_{i}$ es aleatoria. Para comprobar esta relación entre efectos individuales y regresores usamos el test de Hausman $(1978)^{18}$. En la Tabla A3 del Anexo se muestran los resultados de este test. Se observa que no se rechaza la hipótesis nula de no correlación ( $p$-value $=0.45$ ), por lo que se concluye que la mejor estimación es mediante un modelo de efectos aleatorios. De esta forma, el modelo que finalmente estimaremos, controlando por efectos aleatorios, vendrá dado por la siguiente expresión:

$$
\log \left(\frac{S_{i t}}{1-S_{i t}}\right)=\alpha+\delta_{i}+\beta X_{i t}^{\prime}+\lambda_{t}+\varepsilon_{i t}
$$

Con la transformación logística la variable dependiente (nivel de subcontratación) se denomina LTSUBTOT. En la Tabla A3 del Anexo se muestran los resultados y en la Tabla A4 del Anexo se entrega el set

${ }^{17}$ Nivel donde parte el agua subterránea y que puede aprovecharse por medio de pozos.

${ }^{18}$ Véase W. H. Greene (1998), p. 548. 


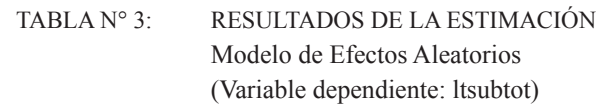

\begin{tabular}{|c|c|c|c|c|c|c|}
\hline \multirow{2}{*}{$\begin{array}{l}\text { Variables } \\
\text { Tipo_oxslf }\end{array}$} & \multirow{2}{*}{$\begin{array}{c}\text { Coef. } \\
-0.109920\end{array}$} & \multirow{2}{*}{$\begin{array}{c}\begin{array}{c}\text { Robust } \\
\text { Error Std. }\end{array} \\
0.242177\end{array}$} & \multirow{2}{*}{$\begin{array}{c}\mathrm{z} \\
-0.45\end{array}$} & \multirow{2}{*}{$\begin{array}{c}\mathrm{P}>\mathrm{Z} \\
0.6500\end{array}$} & \multicolumn{2}{|c|}{ [95\% Intervalo de Conf.] } \\
\hline & & & & & -0.584577 & 0.364738 \\
\hline Dprecior & 0.005410 & $0.002071 * *$ & 2.61 & 0.0090 & 0.001350 & 0.009470 \\
\hline Presionr & 0.010269 & $0.003553 * *$ & 2.89 & 0.0040 & 0.003306 & 0.017232 \\
\hline Tratado_lag & 0.000584 & $0.000261^{*}$ & 2.24 & 0.0250 & 0.000072 & 0.001096 \\
\hline Razonw & -0.060500 & 0.040367 & -1.5 & 0.1340 & -0.139619 & 0.018618 \\
\hline Tasa_acc & -0.044730 & 0.026461 & -1.69 & 0.0910 & -0.096592 & 0.007133 \\
\hline Dep & 0.114873 & 0.271817 & 0.42 & 0.6730 & -0.417879 & 0.647625 \\
\hline Cons & 1.083463 & $0.323349 * * *$ & 3.35 & 0.0010 & 0.449710 & 1.717216 \\
\hline \multirow{4}{*}{$\begin{array}{l}\mathrm{N}^{\circ} \text { de Obs: } 99 \\
\text { sigma_u } \\
\text { sigma_e } \\
\text { rho }\end{array}$} & \multicolumn{3}{|c|}{$\mathrm{N}^{\circ}$ de Grupos: 18} & & & \\
\hline & 0.223427 & & & & R-sq: within & 0.0680 \\
\hline & 0.384635 & & & & between & 0.7458 \\
\hline & 0.252293 & (fraction of & ariance & e to u_i) & overall & 0.4331 \\
\hline
\end{tabular}

Nota: $*$ Significativo al $\mathrm{p}<0.05 ; * *$ significativo al $\mathrm{p}<0.01 ; * * *$ significativo al $\mathrm{p}<0.001$.

completo de parámetros. Se desprende que el nivel de subcontratación aumenta a medida que aumentan el precio del cobre, la presión de los costos y la cantidad de mineral tratado. Estos resultados, de acuerdo a lo esperado, refuerzan las hipótesis de incertidumbre y tamaño como factores determinantes de la subcontratación en la industria minera en Chile. Por otro lado, la especificidad, la diferencia salarial entre ambos grupos (propios y subcontratados), el riesgo de la faena y la propiedad no exhiben efectos significativos sobre el nivel de subcontratación.

\section{Conclusiones}

Este trabajo analiza las causas de la subcontratación en la minería del cobre en Chile. Se usan datos de panel para 18 faenas en el período 2003-2008. Se construye una serie de hipótesis basadas en la literatura previa, las que se testean con el modelo.

Se concluye que en la medida que el precio se desvíe positivamente de su tendencia, es decir, mientras mayor sea el precio del cobre, 
las compañías tienden a intensificar la subcontratación. Una posible explicación para este fenómeno proviene del hecho de que cuando los precios están altos, las faenas desean aprovechar los beneficios derivados de ello, y como el factor variable en el corto plazo es el trabajo, tienden a intensificar el uso de la mano de obra, en particular la flexible.

También se concluye que las presiones de costos son importantes, es decir, a medida que los costos son más altos y la brecha entre los precios y los costos se acorta, las empresas mineras tienden a utilizar más trabajadores flexibles, lo que intensifica los niveles de subcontratación. Esto se explicaría porque a mayores costos, mayor incertidumbre y probabilidades de cierre, por lo que a las firmas les conviene tener más mano de obra flexible que permanente, pues al tener contratos a plazo fijo, los costos involucrados son menores.

Asimismo, se observa que el tamaño de las faenas es importante. Éste se mide como el cociente entre la producción y la ley del yacimiento, lo que sería una proxy del mineral tratado. Se concluye que a mayor tamaño, mayor el número de subcontratados. La posibilidad de concentrarse en aquellas actividades más ligadas al giro propio de la faena minera, más estratégicas y que tienden a aumentar el valor de la compañía explicarían esta relación. Aun más, en la medida que una firma crece, se forman relaciones más complejas, aumenta el costo de monitorear, por lo que lo más conveniente sería subcontratar.

La especificidad de las faenas se mide a través de la proporción de producción vía óxidos y sulfuros. Del análisis se concluye que no se observan efectos significativos de esta variable sobre el nivel de subcontratación. Existen al menos dos explicaciones para este resultado. En primer lugar, podemos suponer que la variable usada como proxy no mide especificidad. Idealmente nos habría gustado contar con información sobre el tipo de trabajador y la tarea desarrollada por cada trabajador, sin embargo estos datos no están disponibles. En lugar de eso, intentamos capturar este efecto asumiendo que los distintos procesos de producción requieren distintos niveles de uso de activos específicos. Segundo, aun cuando este supuesto esté correcto (esto es, la variable escogida efectivamente sea una buena proxy), su efecto puede ser nulo, ya que la especificidad del proceso productivo no trae apareado un problema de oportunismo (hold-up). A pesar de los resultados, no podemos 
decir que la industria de la minería en Chile no se vea enfrentada a problemas de oportunismo ${ }^{19}$.

Los costos laborales de la faena no resultan significativos, lo que indica que si bien existe un diferencial de salarios entre los trabajadores propios y los subcontratados - estos últimos ganan menos-, esta diferencia no explica el aumento en el número de trabajadores externos. Es decir, las compañías no han tendido a aumentar la subcontratación como una forma de ahorrar costos sino que, como se desprende del estudio, existe un conjunto de otras variables que explican de manera importante esta situación. Algo similar ocurre con el riesgo de las faenas, donde de existir alguna relación, ésta sería negativa. Es decir, no porque una determinada faena sea riesgosa, las empresas mineras tienden a intensificar el uso de mano de obra flexible, muy por el contrario, la tienden a disminuir. Una eventual explicación para este comportamiento de las firmas mineras podría ser que aquellas secciones más riesgosas de la mina estarían siendo sustituidas por capital. Este análisis debiera ser ampliado, para incluir efectos no contemporáneos de los salarios sobre los niveles de subcontratación.

Por último, en cuanto a la propiedad, ésta no arroja resultados significativos, indicando que las faenas de Codelco no exhiben mayores tasas de trabajadores subcontratados que las firmas privadas, controlando por el resto de los factores.

Consideramos este trabajo como un primer intento en ahondar los determinantes de la subcontratación en la minería chilena. Creemos necesario continuar esta discusión y seguir desarrollando análisis en el tema que puedan sostener o rebatir las conclusiones aquí alcanzadas. La mayor disponibilidad de datos y alternativas para desarrollar un trabajo empírico contribuirán a lograr un conocimiento más acabado de un tema de primera relevancia para el país.

${ }^{19}$ De hecho, los conflictos protagonizados por los subcontratistas en el pasado pueden ser vistos como evidencia de que ellos tienen algún grado de poder de negociación, reflejando la existencia de oportunismo. Sin embargo, el problema no surge necesariamente a partir de la especificidad (trabajadores desarrollando tareas clave), sino a partir de un problema de números (alto porcentaje de trabajadores subcontratados versus trabajadores propios). 


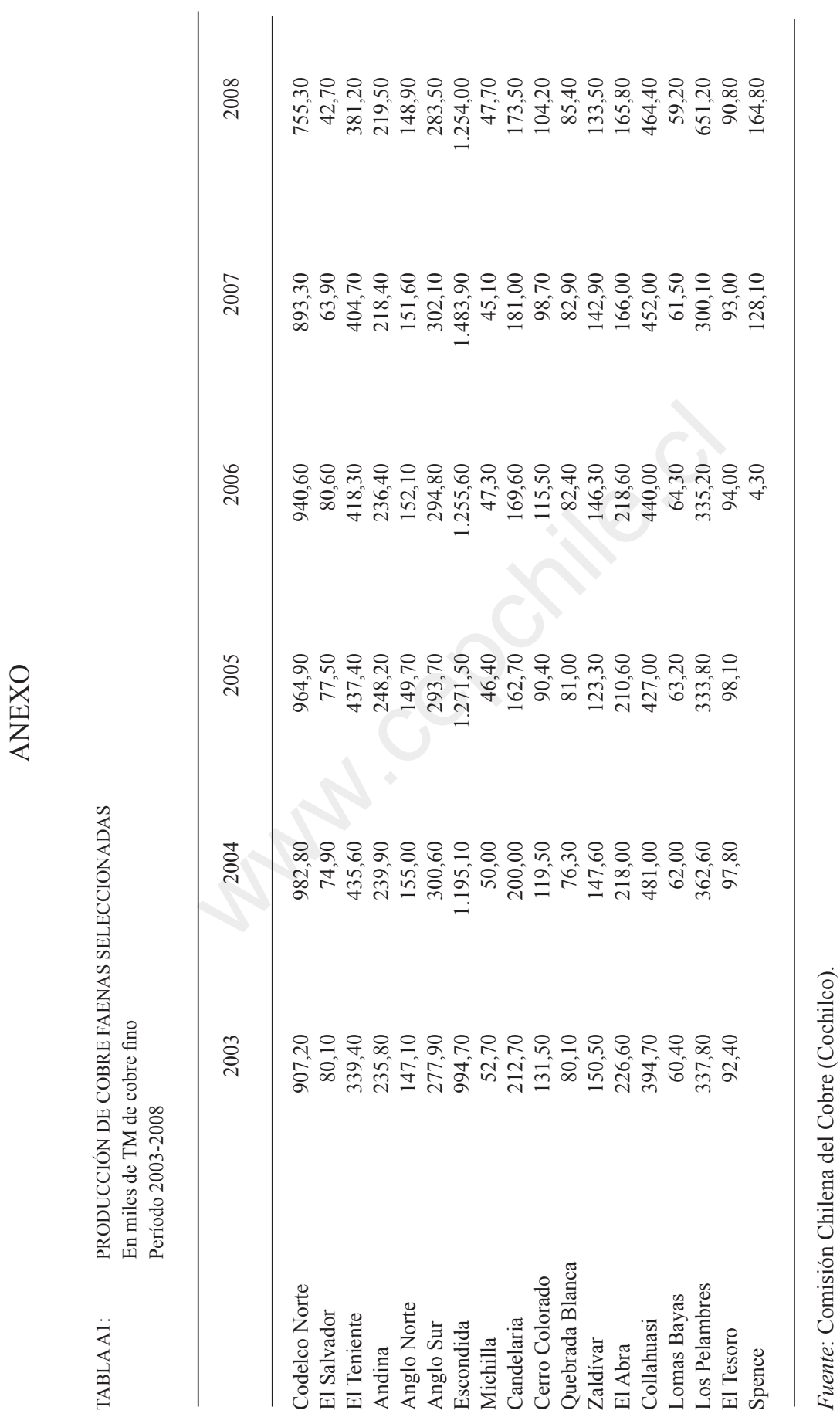




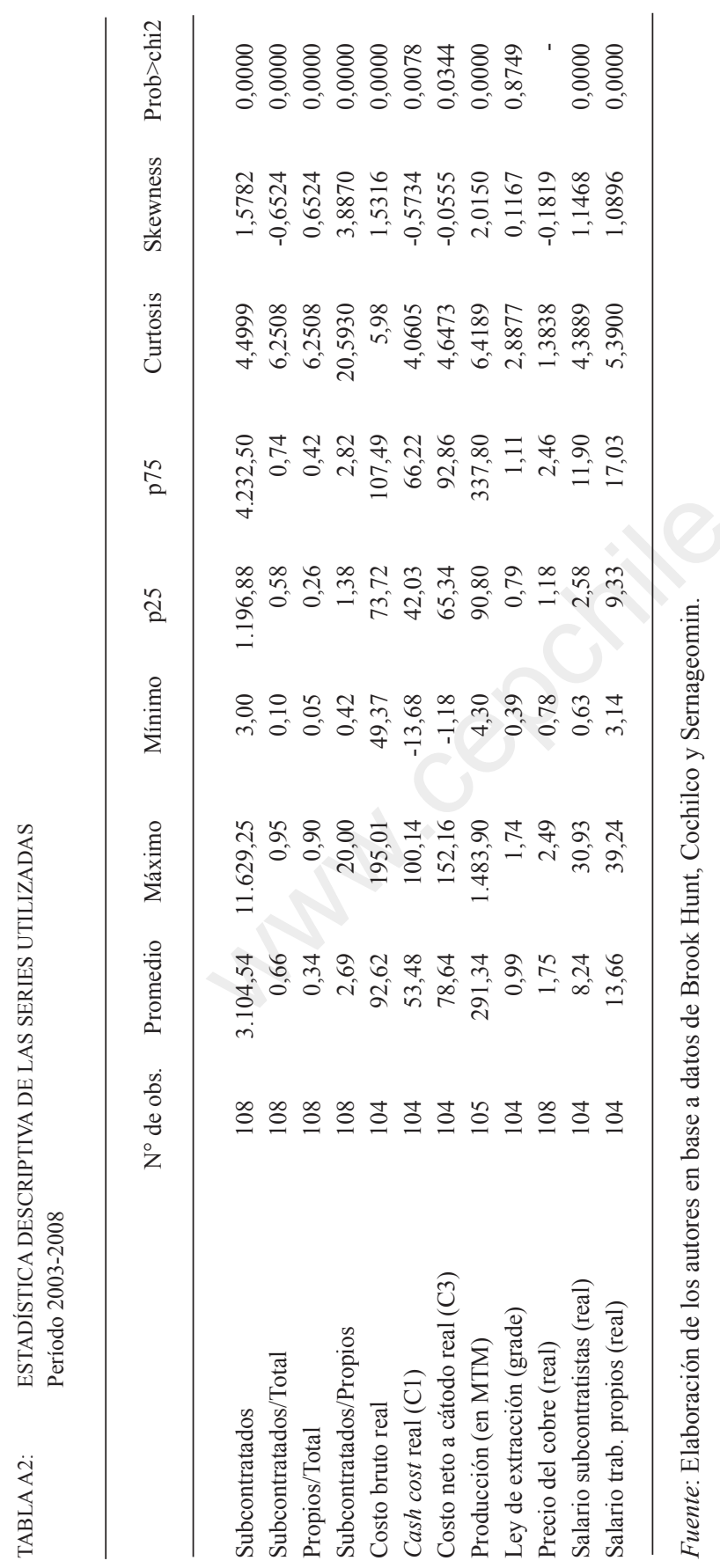




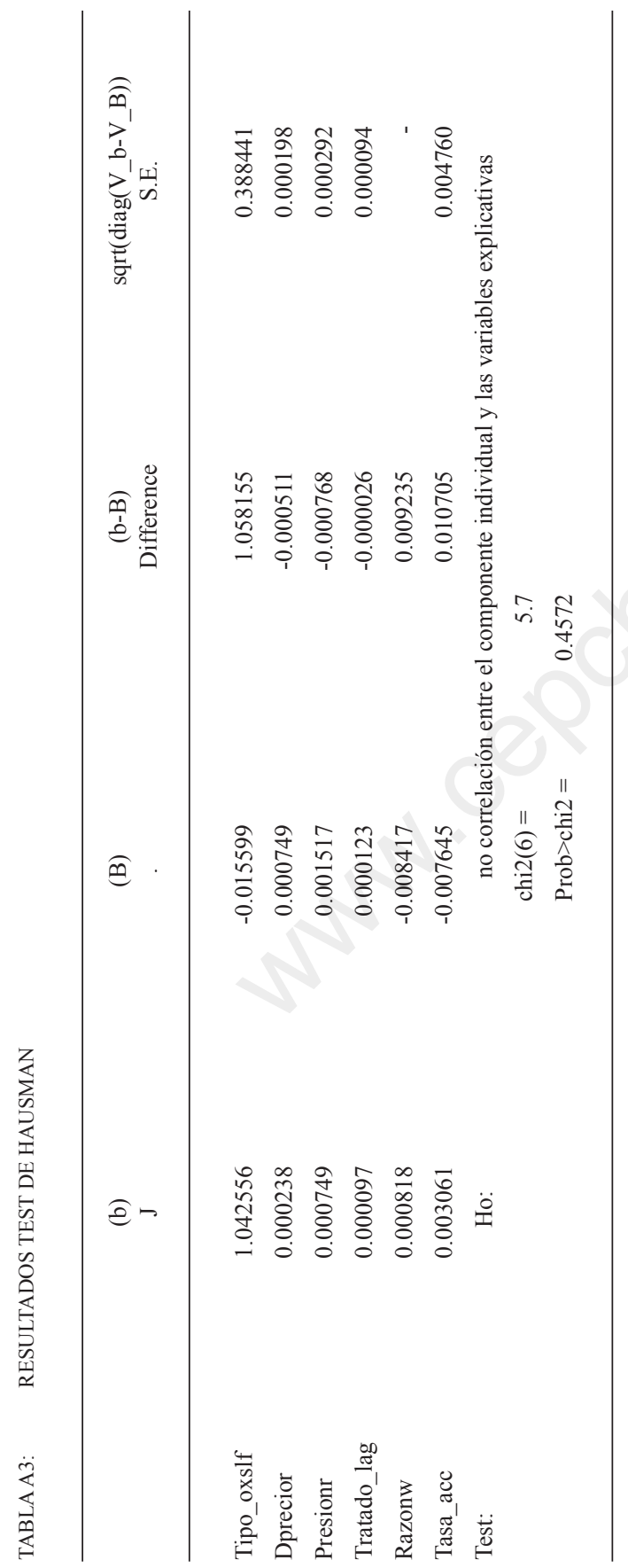




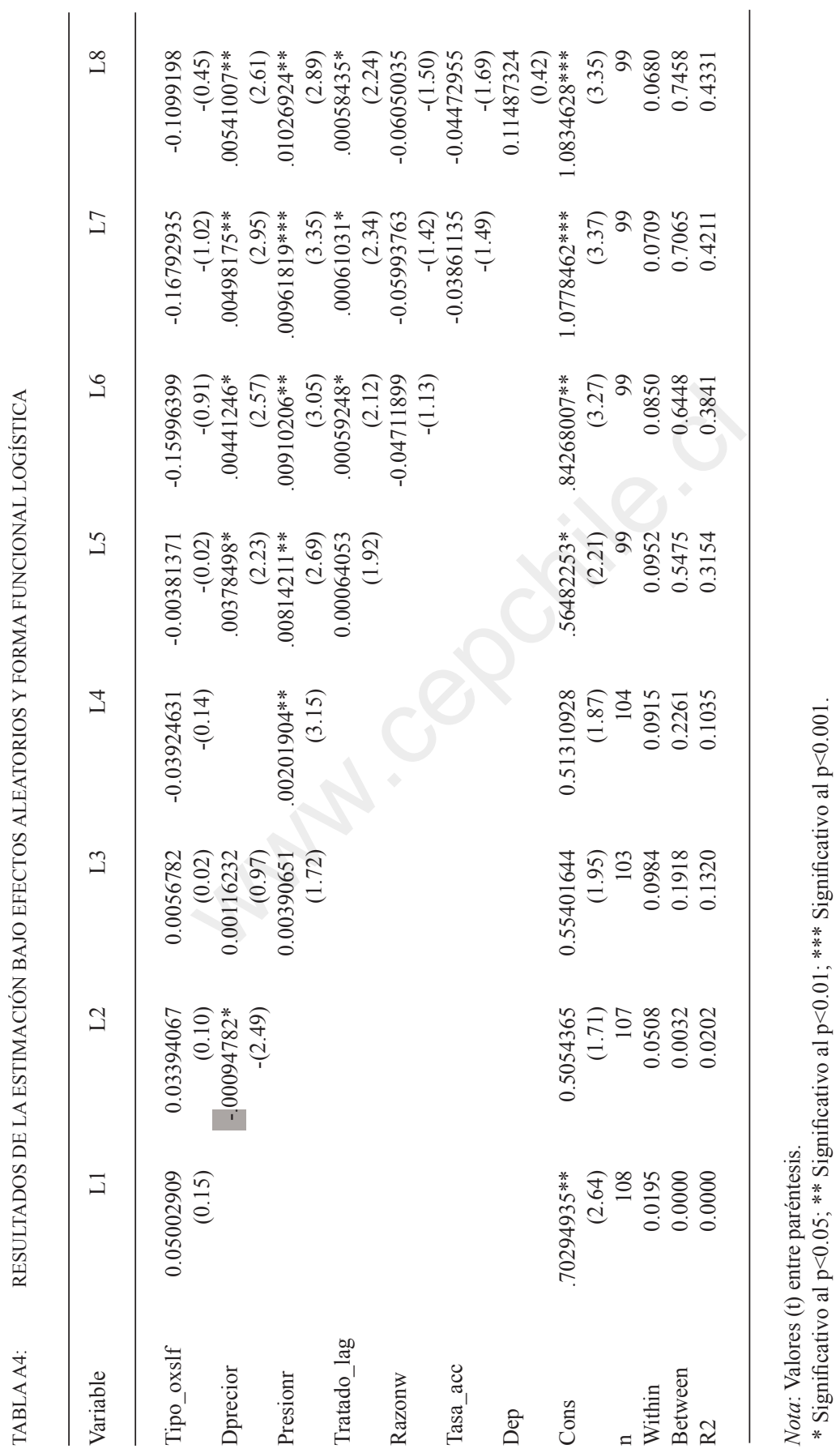




\section{REFERENCIAS}

Abraham, K. G. (1988). "Flexible Staffing Arrangements and Employers' Short-Term Adjustment Strategies". NBER Working Paper Series № 2617.

Abraham, K. G. y S. K. Taylor (1996). "Firms' Use of Outside Contractors: Theory and Evidence". Journal of Labor Economics, Vol. 14, № 3, pp. 394-424.

Alexander, M. y D. Young (1996). "Outsourcing: Where's the Value?" Long Range Planning, Vol. 29, $\mathrm{N}^{\circ}$ 5, pp. 728-730.

Bryce, D. y M. Useem (1998). "The Impact of Corporate Outsourcing on Company Value". European Management Journal, Vol. 16, № 6, pp. 635-643.

Coase, R. (1937). "The Nature of the Firm". Economica, New Series, Vol. 4, № 16, pp. 386-405.

Daroch, S. (2008). "Los Conflictos Laborales en Chile: Principales Ejes para la Discusión". Estudios del Consejo Asesor Presidencial para el Trabajo y la Equidad.

Davis-Blake, A. y B. Uzzi (1993). "Determinants of Employment Externalization: A Study of Temporary Workers and Independent Contractors". Administrative Science Quarterly, Vol. 38, N² 2, pp. 195-223.

Díaz J., R. Lüders y G. Wagner (2006). “La República en Cifras: 1810-2000”. Mimeo, Departamento de Economía, Pontificia Universidad Católica de Chile.

Echeverría, M. (1997): "Subcontratación de la Producción y Subcontratación del Trabajo", Temas Laborales N 7, Dirección del Trabajo. - (2006): "Los Riesgos Laborales de la Subcontratación", Serie Aporte al Debate Laboral № 19, Departamento de Estudios, Dirección del Trabajo.

Echeverría, M., V. Solis, J. Ugarte y V. Uribe-Echevarría (2001). "El Otro Trabajo: El Suministro de Personas en las Empresas". Cuaderno de Investigación $\mathrm{N}^{\circ} 7$ (Reedición), Departamento de Estudios, Dirección del Trabajo.

Escobar, P. y D. López (1996). "El Sector Forestal en Chile: Crecimiento y Precarización del Empleo". Colección de Estudios Sectoriales № 12, Programa de Economía del Trabajo.

Gibbons, R. (2005). "Four Formal(izable) Theories of the Firm?". Journal of Economic Behavior and Organization, Vol. 58, $\mathrm{N}^{\circ} 2$, pp. 200-245.

González, M., B. Arruñada y A. Fernández (2000). "Causes of Subcontracting: Evidence from Panel Data on Construction Firms". Journal of Economic Behavior and Organization, Vol. 42, № 2, pp. 167-187.

Gramm, C. L. y J. F. Schnell (2001). "The Use of Flexible Staffing Arrangements in Core Production Jobs". Industrial and Labor Relations Review, Vol. 54, № 2, pp. 245-258.

Greene, W. H. (1998). Análisis Econométrico. Prentice Hall: Tercera Edición.

Grossman, S. J., y O. D. Hart (1986). "The Costs and Benefits of Ownership: A Theory of Vertical Integration". Journal of Political Economy, Vol. 94, pp. 691-719.

Hanson, G. H. (1995). "Incomplete Contracts, Risk, and Ownership". International Economic Review, Vol. 36, N² 2, pp. 341-363.

Hausman, J. (1978). "Specification Tests in Econometrics". Econometrica, Vol. 46, N 6, pp. 1251-1271.

Houseman, S. N. (1997). "Temporary, Part-Time, and Contract Employment in the United States: A Report on the W. E. Upjohn Institute's Employer Survey on 
Flexible Staffing Policies”. Final Report, U. S. Department of Labor, Office of the Assistant Secretary for Policy.

Joscow, P. L. (2005). "Vertical Integration". En Claude Mènard y Mary M. Shirley (eds.), Handbook of New Institutional Economics. Springer.

Klein, B., R. G. Crawford y A. A. Alchian (1978). "Vertical Integration, Appropriable Rents, and the Competitive Contracting Process". Journal of Law and Economics, Vol. 21, pp. 297-326.

López, D. (2008). Subcontratación y Conflictos Laborales: Un Creciente Descontento en el Trabajo. Santiago: Universidad Central.

Silva, C. (2007). "La Subcontratación en Chile: Aproximación Sectorial". Consejo Asesor Presidencial Trabajo y Equidad, diciembre de 2007.

Stigler, G. J. (1951). "The Division of Labor I Limited by the Extent of the Market". Journal of Political Economy, Vol. 59, $\mathrm{N}^{\circ} 3$, pp. 185-193.

Teece, D. J. (1986). "Profiting from Technological Innovation: Implications for Integration, Collaboration, Licensing and Public Policy". Research Policy, Vol. $15, \mathrm{~N}^{\circ} 6$, pp. 285-305.

Williamson, O. E. (1971). "The Vertical Integration of Production: Market Failures Considerations". American Economic Review, Vol. 61, № 2, pp. 233-261. (1975). Markets and Hierarchies: Analysis and Antitrust Implications. New York: Free Press.

(1979): "Transaction Costs Economics: The Governance of Contractual Relations". Journal of Law and Economics, Vol. 22, pp. 233-262. (1985). The Economic Institutions of Capitalism. New York: Free Press.

Recibido: octubre de 2009. Aceptado: julio de 2010. 